\title{
Light Environmental Management for Artificial Protected Horticulture
}

\section{Wenke Liu',2*}

${ }^{1}$ Institute of Environment and Sustainable Development in Agriculture, Chinese Academy of Agricultural Sciences, Beijing 100081, China ${ }^{2}$ Key Labarotary of Energy Conservation and Waste Management of Agricultural Structures, Ministry of Agriculture, Beijing 100081, China

\begin{abstract}
Light is a vital environmental factor that affects plant growth and development by acting on plants not only as the sole energy source of photosynthesis, but also as the kind of external signal. Light requirements of plants are subjected to species, cultivar, growth and developmental stages of plant, environmental conditions and manipulation target of yield \& quality. Therefore, detailed studies on light formula (LF) based on physiological requirement are urgently needed for getting high yield and good quality in plants. With the development of semiconductor solid light sources, light-emitting diodes (LEDs), light quality physiology and light formula in plants have been gradually and extensively conducted worldwide by taking advantage of LED providing precise light spectrum and close illumination. LF defined as optimized and integrated assembly of spectral component emitting from light sources (particularly LEDs) that is suitable for plant productivity and nutritional quality formation. Based on published literatures, red, blue, and compound white light are macro-necessary light spectrum, and purple, green, yellow and orange light are micro-beneficial light spectrum, while far-red light and ultraviolet light are beneficial light spectrum. However, other light spectrum except photosynthetic active radiation, far-red and ultraviolet light is invalid for plant cultivation. Our hypothesis is that one or two kinds of necessary light quality, special micro-beneficial and beneficial light quality can make up a light formula for a kind of plant during certain period and certain environmental conditions. LF is a crucial scientific issue that should be established for protected cultivation plants with artificial light or plants that needed supplemental light. More importantly, LF is an important part of light environment management strategy (LEMS), referring to management of light intensity, LF and photoperiod. LEMS should be established for plant cultivation with artificial light sources. In this project, the application of LF and LEMS will be used in the plant factory for the advantages of entire artificial light source, cultivation with nutrient solution and intelligentized environmental control. Furthermore, vegetables, particularly leafy vegetables, will be preferentially used for the project. To sum up, the study will focus on LF which will provide technique parameters for potential efficiency (high yield and good quality) during plant growth and development.
\end{abstract}

Keywords: Light formula; Light quality fomula; Necessary light spectrum; Light-Emitting Diodes (LEDs); Plant factory; Vegetable

\section{Introduction}

Protected vegetable cultivation has developed rapidly worldwide to meet the increasing demand for fresh vegetables. For example, it was estimated that the planting area of China of protected vegetables reached four million hectares in 2010 [1]. Nowadays, the protected facilities mainly include glasshouse, greenhouse, Chinese solar-greenhouse, plastic tents and plant factories for off-season vegetable cultivation. Plant factory is the top pattern of modern protected horticulture. Currently, dozens of plant factories, especially vegetable factories, are in operation in some countries, e.g. Japan, China, and Netherlands etc. As the dominant type, the vegetable factories trace their roots back to the Christensen Farm in Denmark, where production first started in 1957 [2].

Light is a vital environmental factor that affects plant growth and development by acting on plants not only as the sole energy source of photosynthesis (acting on chlorophyll), but also as the kind of external signal (acting on cryptochrome, phototropin and the other photoreceptors) after being intercepted and absorbed by photosynthetic tissue. It is well known that plant growth and development are regulated by light quality, light intensity and photoperiod, so above three elements are key components of light condition. Under cover, besides photosynthetically active radiation (PAR) (400-700 nm), the medium-wave ultraviolet light (UV-B, 280-320 nm) long-wave ultraviolet light (UV-A, 320-400 nm) are important spectral components for vegetable production. However, due to the absorption and obstruction effects of cover materials (glass and plastic film), only about $88 \%$ visible light and $15.9-21.1 \%$ ultravio- let irradiation is transmitted into protected systems, which resulted in significant reduction in light intensity and even substantially modification in light spectrum composition [3-5]. In order to obtain an optimal light environment for vegetable growth, artificial light is needed to overcome the irregular diurnal light intensity changes of natural light in greenhouse or as the sole main light source. More importantly, UV-A, UV-B and UV-C irradiation levels in closed cultivation systems with artificial light, e.g. plant factory, are very low, since ultraviolet irradiation is not present in fluorescent lamps and light-emitting diodes (400-700 $\mathrm{nm}$ ). Therefore, detailed investigation on effects of light quality of PAR and ultraviolet irradiation on growth, physiological metabolisms and nutritional quality is necessary.

Generally, the improvement of yield and nutritional quality of vegetables in protected facilities is the final target of light environment regulation. Based on current literatures, precise spatiotemporal management of light conditions (light quality, light intensity and photoperiod) can enhance yield and accumulation of health-beneficial phytochemicals in vegetables. Optimized light environment regulation can

*Corresponding author: Dr. Wenke Liu, Institute of Environment and Sustainable Development in Agriculture, Chinese Academy of Agricultural Sciences, Beijing 100081, China, E-mail: liuwke@163.com

Received May 16, 2012; Accepted May 19, 2012; Published May 21, 2012

Citation: Liu W (2012) Light Environmental Management for Artificial Protected Horticulture. Agrotechnol 1:101. doi:10.4172/2168-9881.1000101

Copyright: (c) 2012 Liu W. This is an open-access article distributed under the terms of the Creative Commons Attribution License, which permits unrestricted use, distribution, and reproduction in any medium, provided the original author and source are credited. 
be environmentally friendly. High-quality vegetables can be produced in protected facilities. Liu et al. [6] defined high-quality vegetables as vegetables not containing over-the-limit harmful substances (nitrate, nitrite, heavy metals and pesticides), and not having negative effects on human health after ingestion. Health-beneficial phytochemicals of vegetables are referred to some substances, such as ascorbic acid, carotenoids, phenolics, flavoids, which are influential beneficially to human health.

In greenhouses, the artificial light source is used to support the natural light, while the artificial light source itself entirely determines the light conditions in closed vegetable factories. So, the strategy for balancing the light requirements of vegetables to the light conditions is largely different in the two kinds of protected facilities. Comparatively, the latter is relatively easy to realize. In our view, it is possible to regulate the productivity and nutritional quality of horticultural crops in protected facilities. However, what kind of light quality or wavelength combinations is suitable for a specific horticultural crop? How to manage light quality, light intensity and photoperiod synergically? What is the light environment management strategy for various protected facilities? These issues are far fully investigated, understood and answered. Therefore, more work is urgently needed to clarify the relationship between light conditions (light quality, light intensity and photoperiod) and growth \& quality at every developmental stages of plants in various protected systems. Apparently, closed plant factory with artificial lighting is the ideal protected facility as objective that should be studied on above issues preferentially, and study outcome is priorily applied in closed plant factory.

\section{Light Formula (LF): Concept and Significance}

As qualitative factor, light quality is the primary component in light condition. Based on current literatures, biologically active light quality can be classified into three zones located in spectrum, including PAR zone $(400 \mathrm{~nm}$ to $700 \mathrm{~nm})$, far-red zone $(700 \mathrm{~nm}$ to $750 \mathrm{~nm})$, and ultraviolet irradiation zone (200 nm t0 $400 \mathrm{~nm}$ ). PAR are comprised of red, blue, green, purple, yellow, orange, cyan, while ultraviolet irradiation is comprised of UV-A (320 nm to $400 \mathrm{~nm}), \mathrm{UV}-\mathrm{B}(280 \mathrm{~nm}$ to $320 \mathrm{~nm})$, and UV-C (200 nm to $280 \mathrm{~nm}$ ). Higher plants are empowered with an array of photoreceptors controlling diverse responses to light quality for plant growth and its photo morphogenesis. Generally, plants have three different photoreactions: (1) red and blue light used in photosynthesis; (2) blue light used in cryptochrome and phototropin reaction systems; (3) red and far-red light enabling reversible switching of the phytochrome system [7].

For natural light, light quality is often unsuitable for horticultural growth and health-beneficial phytochemical formation. Traditionally, both supplemental lighting in greenhouse and in closed vegetable factories, use stable light conditions to maintain photosynthesis and photo morphogenesis, so light is a critical yet passive entity. With the development of light-emitting diode (LED), the potential to actively implement dynamic lighting strategies to control plant growth and development, and nutritional quality holds great promise in the future of protected cultivation. LEDs can tailor illumination spectra according to plant requirements. Based on the advancements in LED illumination technology, here, we put forward light formula (LF) concept for guiding the optimization of light quality application in protected plant cultivation. LF is defined as an optimized light quality component aiming at high productivity or nutritional quality under protected production. Basically, LF of plants are subjected to species, cultivar, growth and developmental stages of plant, environmental conditions and manipulation target of yield \& quality. In other words, LF is cultivar-specific, dynamic and adjustable accompanied with the corresponding biological processes of plants. Not only establishment of plant LF relies on LED technology development, also application of LF relies on LED lighting systems. Surely, LED, a sort of solid-state, narrow bandwidth lighting platforms, offer a unique opportunity to realize light quality precise managements according to LF. In the future, a set of specific LF should be studied and developed for specific horticultural crops, and LF may be adjusted throughout the entire life of plant to potentially optimize traits of interest, such as morphology, yield and nutritional quality.

LF and its management strategy are of great significance for closed plant factory production with artificial light. (1) LF management will save energy by deleting extra light spectra; (2) LF management will maximize the yield and/or nutritional quality; (3) LF management is a basis for establish light environment management strategy (LEMS). Nowadays, LF and its management strategy is far perfectly established. Detailed studies on light formula (LF) based on physiological requirement of plants one by one are urgently needed for high yield and quality targets.

\section{Light-Emitting Diodes (LEDs): An Optimal Tool for LF Research}

LF, in essence, is an optimized and integrated assembly of spectral component emitted from light sources that is suitable for plant productivity and nutritional quality formation. LED is an ideal tool to study light quality requirements of every horticultural crop. Their small size, durability, long operating lifetime, wavelength specificity, relatively cool emitting surfaces, and linear photon output with electrical input current make these solid-state light sources ideal for use in plant lighting designs. Because the output waveband of LEDs is much narrow than that of traditional sources of electric lighting used for plant growth, one challenge in designing an optimum light spectrum [8]. LEDs have tremendous potential as supplemental or sole-source lighting systems for crop production. Several accepted advantages of LED can be classified. First, LED is energy-saving, small size and with high light efficiency; Second, LED is cold light source with low thermal radiation, so it can illuminate plants closely; Third, LED can emit multiple narrow-bandwidth monochromatic light with specific wavelengths, almost covering all biologically active light qualities. In addition, light quality biology and physiology have been gradually and extensively conducted worldwide by taking advantage of LED providing precise light spectrum and close illumination, which provide abundant data to establish LF. Totally, LED is a best candidate as light source for regulating plant biology, productivity and nutritional quality. Up to date, a number of studies and their findings are useful for selection of LED types and positioning for a variety of purposes depending on crop type and desired responses. To select specific wavelengths for a targeted plant response makes LEDs more suitable for plant-based uses than many other light sources. More importantly, compound or mixed light quality according to LF can be realized through combination with various LEDs. Thus, using LED as artificial light source; light quality can be controlled with great precision as desired [9].

\section{Light Formula (LF) for Protected Horticultural Plants: Primary Conclusions}

LEDs have become an optimal tool for LF research of plants, particularly vegetables in closed plant factory or growth chamber since 1990s last century. It is well known that NASA's Kennedy Space Center and Purdue University had made great contributions for LED lighting technology [8,9]. Today, scientists of many countries, e.g. Japan, USA and 
China, are positively working in this field. With rapid development of LED technology in 21th century, research on light biology and physiology of vegetables accelerated because more pure light wavelengths were emitted from novel LED lighting systems.

In 1990s, some initial studies focused on usefulness of LEDs as a sole source or as supplemental lighting for plant growth. For PAR, biological effects of red light plus blue light were extensively investigated on lettuce [10-12], strawberry [13] and pepper [14], wheat [15] and rice [16]. Goins et al. [15] indicated that wheat can complete its life cycle under red LEDs alone, but larger plants and greater amounts of seeds are produced in the presence of red LEDs supplemented with a quantity of blue light. Matsuda et al. [16] also found that rice plants grown under a combination of red $(660 \mathrm{~nm})$ and blue $(470 \mathrm{~nm})$ LEDs sustained higher leaf photosynthetic rates than did leaves from plants grown under red LEDs only. Yorio et al. [12] summarized previous blue light work and reported that yield of lettuce, spinach, and radish crops grown under red LEDs alone was reduced compared with when blue fluorescence was included to give the same final PPF. Schuerger et al. [17] examined changes in leaf anatomy of pepper under different color combinations of light. They used red (660 nm) LEDs combined either with FR (735 $\mathrm{nm}$ ) LEDs or BF lamps at the same PPF. Their results indicated that leaf thickness and number of chloroplasts per cell depended much more on the level of blue light than the red : FR ratio. Many horticultural and grain crops had been cultivated successfully under LED light sources with red light, blue light or red plus blue light quality, including lettuce, spinach, strawberry, tomato, cucumber, potato, wheat and rice as white light. Furthermore, mixed light quality comprised of suitable red light and blue light ratio did facilitate high productivity or nutritional quality, e.g. lettuce [18], spinach [19], strawberry [20]. Previous work has focused on the proportion of blue light required for normal plant growth as well as the optimum wavelength of red and the red/far-red ratio. Thus it can be concluded that, as white light, blue light and red light is necessary light quality for plant cultivation. Combination of red and blue lighting was an effective light source for several crops.

Other light qualities were also evidenced in yield or quality benefits in vegetable production under artificial lighting. Kim et al. [21] found that the addition of $24 \%$ green light to red and blue LEDs enhanced lettuce growth since green light can better penetrate the plant canopy and potentially increase plant growth by increasing photosynthesis. The addition of green light could offer more benefits, since green light can better penetrate the plant canopy and potentially increase plant growth by increasing photosynthesis from the lower canopy leaves. Similarly, other light wavelengths, such as yellow, orange, purple, cyan and so on, may affect horticultural crops to some extent under certain conditions. This needs further investigations. Liu et al. [22,23] plants under red+blue and red+blue+green light were significantly stronger and shorter, while under yellow, green and red light were weaker and higher compared with the white light treatment. Photosynthetic pigments were shown to have significant difference under respective light irradiations of LEDs. Taken together, red plus blue and red plus blue and green light of LEDs were shown to be beneficial factors for the growth and photosynthesis of cherry tomato seedlings.

Recently, usefulness of supplemental ultraviolet radiation on growth and nutritional quality of protected vegetables were also explored. Using UV-A, blue, green, red, and far-red LEDs, authors investigated the effects of different supplemental light qualities on phytochemicals and growth of baby leaf lettuce, grown white fluorescent lamps as the main light source inside a growth chamber [24]. Briefly, supplemental blue or UV-A could enhance accumulation of anthocyanins, supplemental blue also increased carotenoids concentration, supplemental $\mathrm{R}$ could increase phenolics concentration while supplemental far-red light could increase biomass, but result in lower phytochemical concentrations. A controlled light quality with an appropriate ratio of blue, red or far-red light quality provided as supplemental light may improve phytochemical content and biomass of plants grown under white light. Further studies are needed to describe the effects of different ratios of selected light qualities in both growth chamber and greenhouse conditions. The results demonstrated that supplemental light quality could be strategically used to enhance nutritional value and growth of baby leaf lettuce grown under white light [24]. Via continuous lighting by LED, the contribution of red light to significant $\beta$-carotene expression and antioxidant activity for nutrition and health benefits are emphasized [25].

Also, the potential for increasing secondary compounds in vegetables by using supplemental selected UV irradiation have been extensively investigated [26,27]. UV radiation can be regarded as a stress factor which is capable of significantly affecting plant growth characteristics. Generally, plant height, leaf area, leaf length have been showed to decrease, whereas leaf thickness was increased in response to UV-B radiation [28]. Plants produce a wide range of flavonoids and related phenolic compounds which tend to accumulate in leaves of higher plants in response to UV radiation [28]. Plants may produce secondary products to protect them against UV light damage, but these metabolites also play an important role in human health. Phenolics, flavonoids and anthocyanins are responsible for antioxidant activity in fruits and vegetables [29].

Based on published literatures, red, blue, and compound white light are macro-necessary light spectrum, and purple, green, yellow and orange light are micro-beneficial light spectrum, while far-red light and ultraviolet light are beneficial light spectrum. Spectral changes of illumination evoked different morphogenetic and photosynthetic responses, which can vary among different plant species. However, other light spectrum except photosynthetic active radiation, far-red and ultraviolet light is invalid for plant cultivation. Our hypothesis is that one or two kind of necessary light quality, special micro-beneficial and beneficial light quality can make up a light formula for a kind of plant during certain period and certain environmental conditions. LF is a crucial scientific issue that should be established for protected cultivation plants with artificial light or plants that needed supplemental light.

\section{LF: An Important Part of the Light Environment Man- agement Strategy (LEMS)}

Establishment of LF will lay a foundation for establishing light environment management strategy (LEMS) for given protected plant species cultivated with artificial light source. Besides LF, light intensity and photoperiod are key contents of the light environment management. The LEMS refers to a comprehensive management method of light environment, including LF, light intensity and photoperiod for entire life of one special plant species, which should be established to facilitate high productivity and good nutritional quality with artificial LED light sources. By using LED lighting systems, it is possible to continually adjust fluence rate, wavelength combinations, and photoperiods to actively manipulate plant morphology and production, rather than using a stable light condition traditionally.

The application of LEMS will be used in the plant factory for the advantages of entire artificial light source, cultivation with nutrient solution and intelligent environmental control. Furthermore, vegetable production, particularly leafy vegetable cultivation, will benefit pref- 
erentially from the use of LEMS in plant factory. Corresponding LED lighting systems for plant factory should be designed and developed. Ideally, for an optimum plant production and product quality, light environment have to be adapted to the needs of the plants at every moment controlled by LEMS.

\section{Conclusions}

With the development of LED illumination technology, light biology and physiology of protected horticultural crops were conducted extensively. Simultaneously, LEDs are bringing a breakthrough in artificial lighting in protected horticulture. LEDs provide a tool to facilitate the study and establishment of the LF and LEMS of every target horticultural crops. In return, LF will provide technique parameters for design of species-specific lighting regimes may help maximize plant productivity and benefits of food quality. Optimization of lighting system, establishment of LF and LEMS will drive plant cultivation in plant factory greatly either in productivity or nutritional quality.

\section{References}

1. Zhang ZB (2011) Development countermeasure of protected vegetables with low carbon production technology. In: Yang QC, Kozai T, Bot GPA. Protected Horticulture Advances and Innovations-Proceedings of 2011 the $2^{\text {nd }}$ High-level International Forum on Protected Horticulture (Shouguang •China). China Agricultural Science and Technology Press. Beijing. 9-13.

2. Takatsuji M (1986) Vegetable plant factory. Maruzen, Tokyo.

3. Nitz GM, Grubmuller E, Schnitzler WH (2004) Differential Flavonoid Response to PAR and UV-B Light in Chive (Allium schoenoprasum L.).Acta Hortic 659: 825-830

4. Chen L, Wu Z (2008) Effects of ultraviolet-B radiation on growth,yield and quality of pakchoi(Brassica campestris ssp.chinensis). Journal of Plant Resources and Environment 17: 43-47.

5. Peng Y, Ai X (2010) A review on effects of UV-B increase on vegetables. Modern Horticulture 6: 16-17.

6. Liu WK, Yang QC, Du LF (2009) Soilless cultivation for high-quality vegetables with biogas manure in China: feasibility and benefit analysis. Renewable Agriculture and Food Systems 24: 300-307.

7. Watanabe $H$ (2011) Light-controlled plant cultivation system in Japan-development of a vegetable factory using LEDs as a light source for plants. Acta Hortic 907: 37-44.

8. Massa GD, Kim HH, Wheeler RM, Mitchell CA (2008) Plant productivity in response to LED lighting. Hort Science 43: 1951-1956.

9. Folta K M, Childers KS (2008) Light as a growth regulator: controlling plant biology with narrow-bandwidth solid-state lighting systems. Hort Science 43 : 1957-1964.

10. Bula RJ, Morrow RC, Tibbitts TW, Barta DJ, Ignatius RW, et al. (1991) Lightemitting diodes as a radiation source for plants. Hort Science 26: 203-205.

11. Yanagi T, Okamoto K, Takita S (1996) Effects of blue, red, and blue/red lights of two different PPF levels on growth and morphogenesis of lettuce plants. Acta Hortic 440: 117-122.

12. Yorio NC, Goins GD, Kagie HR, Wheeler RM, Sager JC, et al. (2001) Improving spinach, radish, and lettuce growth under red light-emitting diodes (LEDs) with blue light supplementation. Hort Science 36: 380-383.

13. Yanagi T, Okamoto K, Takita S (1996) Effect of blue and red light intensity on photosynthetic rate of strawberry leaves. Acta Hortic : 371-376.

14. Brown CS, Schuerger AC, Sager JC (1995) Growth and photomorphogenesis of pepper plants under red light-emitting diodes with supplemental blue or farred lighting. J Am Soc Hortic Sci 120: 808-813.

15. Goins GD, Yorio NC, Sanwo MM, Brown CS (1997) Photomorphogenesis photosynthesis, and seed yield of wheat plants grown under red light-emitting diodes (LEDs) with and without supplemental blue lighting. J Exp Bot 48:14071413.

16. Matsuda R, Ohashi-Kaneko K, Fujiwara K, Goto E, Kurata K, et al. (2004) Pho- tosynthetic characteristics of rice leaves grown under red light with or without supplemental blue light. Plant Cell Physiol 45:1870-1874

17. Schuerger AC, Brown CS, Stryjewski EC (1997) Anatomical features of pepper plants (Capsicum annuum L.) grown under red light-emitting diodes supplemented with blue or far-red light. Ann Bot 79: 273-282.

18. Zhou WL, Liu WK, Yang QC (2012) Reducing nitrate concentration in lettuce by elongated lighting delivered by red and blue LEDs before harvest. J Plant Nutr (In press).

19. Ohashi-Kaneko K, Takase M, Kon N, Fujiwara K, Kurata K, et al. (2007) Effect of light quality on growth and vegetable quality in leaf lettuce, spinach and komatsuna . Environment Control in Biology 45: 189-198.

20. Yanagi T, Yachi T, Okuda N, Okamoto K (2006) Light quality of continuous illuminating at night to induce floral initiation of Fragaria chiloensis L.CHI-24-1. Sci Hortic 109: 309-314.

21. Kim HH, Wheeler RM, Sager JC, Goins GD (2004) A comparison of growth and photosynthetic characteristics of lettuce grown under red and blue lightemitting diodes (leds) with and without supplemental green LEDs. Acta Hortic 659: $467-475$.

22. Liu XY, Chang TT, Guo SR, Xu ZG, Li J (2011). Effect of different light quality of led on growth and photosynthetic character in cherry tomato seedling. Acta Hortic 907: 325-330.

23. Liu XiaoY, Guo SR, Xu Z, Jiao XL, Takafumi T (2011) Regulation of chloroplast ultrastructure, cross-section anatomy of leaves and morphology of stomata of cherry tomato by different light irradiations of LEDs. Hortiscience 45: 1-5.

24. Li Q, Kubota C (2009) Effects of supplemental light quality on growth and phytochemicals of baby leaf lettuce. Environ Exp Bot 67: 59-64.

25. Wu MC, Hou CY, Jiang CM, Wang YT, Wang CY, et al. (2007) A novel approach of LED light radiation improves the antioxidant activity of pea seedlings. Food Chemistry 101: 1753-1758

26. Tsormpatsidis E, Henbest RGC, Davis FJ, Battey NH, Hadley P, et al. (2008) UV irradiance as a major influence on growth, development and secondary products of commercial importance in Lollo Rosso lettuce 'Revolution' grown under polyethylene films. Environ Exp Bot 63: 232-239.

27. Voipio I, Autio J (1995) Responses of red-leaved lettuce to light intensity, UV-A radiation and root zone temperature. Acta Hort 399:183-190.

28. Rozema J, van de Staaij J, Björn LO, Caldwell M (1997) UV-B as an environmental factor in plant life: stress and regulation. Trends Ecol Evol 12: 22-28.

29. Cao G, Sofic E, Prior RL (1997) Antioxidant and prooxidant behavior of flavonoids: structure-activity relationships. Free Radic Biol Med 22: 749-760. 